\title{
Gamma-ray astronomy: the last observational frontier
}

ON June 10, 11 and 12 a symposium on "The Context and Status of Gamma Ray Astronomy" was held at the ESRIN laboratory in Frascati. The rationale behind such a symposium under the ESRO banner at the present time derives partially from the forthcoming launch of COS-B (see Nature, 249, 398; 1974); but quite apart from this slightly parochial incentive it turned out that gamma-ray astronomy has indeed reached something of a landmark recently, and is now likely to fullfil some of the promise which it has held for so long.

Certainly throughout the 1970 s we have heard repeatedly that the big breakthrough in gamma-ray astronomy 'is about to be made'. But the difficulties of first detecting any gamma-rays from outside the Solar System and secondly deciding from exactly which direction they are coming have made some of the premature claims ring rather hollow in recent years. One result of these difficulties has been a considerable broadening of the accepted definition of gamma rays in this context; some of the data presented at Frascati would have been equally well suited to a symposium about X-ray astronomy. Such definitions are, however, intrinsically arbitrary, and as long as the active observers know what they mean by gamma rays no harm is likely to result from a broadening of the definition.

Ironically, in spite of the intensive efforts made to detect gamma ray events from outside the Solar System the most impressive data gathered so far have come from a series of satellites designed to monitor events on Earth. These, of course, are the Vela satellites, which are intended to detect radiation from nuclear explosions on Earth. Over the past four years, these satellites have not had the opportunity to discover many terrestrial nuclear explosions; but the same laws of physics apply to nuclear explosions elsewhere in space, where bursts of gamma rays are produced. As a result, the various Vela satellites have marked all of the 30 or so gamma ray bursts which have been recorded so far. And in only a couple of cases was it necessary to
A recent symposium provided an insight not only into the present status of gamma-ray astronomy, but also into the conventions of scientific symposia, as John Gribbin reports.

examine the Vela data in the light of evidence gained from other satellites before the evidence of the bursts became clear.

So gamma-ray astronomy can definitely be said to have cleared the first hurdle, of detecting something definite. The second problem has also been at least partially resolved since the proliferation of satellites equipped to detect gamma-ray bursts has made direction finding, by a kind of astronomical triangulation, a feasible proposition. And this, more or less, is where the Frascati symposium comes in. Other topics were also discussed, covering the gamma-ray background, low energy gamma-ray astronomy, and galactic emission. But to an observer from outside the gamma-ray astronomy family, the bursts and their interpretation provided the dominant interest.

I. B. Strong (Los Alamos) reviewed the observational data concerning the bursts, and other contributors elaborated on specific measurements before F. Pacini (Frascati) reviewed theories relating to their origin. In both branches of the investigation, the pioneering nature of gamma-ray astronomy was clearly apparent-but the effect of this on experimentalists and theoreticians differed greatly.

In a very real sense, gamma-ray astronomy is the last frontie- of observational astronomy. It is now possible to observe every part of the electromagnetic spectrum, and the gammaray region is the last for which practical techniques have been developed. The observers, many of whom have a background in particle physics rather than in astronomy, seem somewhat overawed by this, and perhaps by the long struggle they have had to get hold of any worthwhile data. The result is that each observation seems to be exhaustively analysed and picked over for significance-and the danger, of course, is that too much might thereby be read into what may well be atypical, or even incorrect, data.

The theoreticians, however, have fewer inhibitions. Indeed, a great many theoretical astronomers delight in a situation where there is just enough evidence to make model building worthwhile, but not enough to prove that their favoured model is incorrect. The study of gamma-ray bursts today provides a happy hunting ground for such theoreticians.

The fondness of the observers for smothering detail extended even to the presentation of their contributions at the conference. In the most extreme cases, we were told openly that although only a short paper was being presented, the version in the published proceedings of the conference would be much more 'complete'. That hardly seems fair on participants (some of whom had come from the United States and Japan), let alone on the humble reporter. In addition, many of the observations seemed essentially to duplicate one another, so that we had a stream of speakers standing up and saying much the same things about various events observed by different satellites, and showing graphs which seemed to be essentially interchangeable, allowing for the largish error bars.

It this kind of presentation really necessary? Clearly, each group must present its results and have them published in the proceedings of such a key conference, or the holders of the purse strings will want to know what is going on. But if each group had provided a summary of its data, circulated to participants in advance, and one or two speakers had outlined the implications then I feel sure that the symposium would have been just as fruitful, and perhaps a lot less tedious in parts.

Much the same could be said about most scientific symposia. But in the case of gamma-ray bursts such a streamlining of the proceedings would have been particularly apt, since only about three things are known about the bursts. First, they occur about seven times a year. Second, they are 
very sharply defined in time with perhaps a double structure. And third, on the basis of 12 direction measurements, their distribution seems isotropic.

Of course, much more in the way of detailed measurements is available, and was presented. But that is just about all the evidence on which the theoreticians can build their models. Nothing daunted, the theoreticians have proceeded to do just that, as Pacini described.

The isotropy of the burst distribution provides vital information. Either the bursts originate nearby (a few hundred parsecs from the Sun), or they must be extragalactic. The intermediate case, of a distribution over a large part of our Galaxy, would reveal structure related to the structure of the Galaxy. With that proviso, Pacini happily listed the models which have been proposed: radiation from relativistic dust grains entering the Solar System; comets falling into collapsed stars; traces of "defunct" pulsars; superflares on stars; supernovae in external galaxies; birth of neutron stars; and the "first manifestation" of radio outbursts in external galaxies.

It does not really matter which, if any, of these models may turn out to be correct. What is significant is that theoreticians are prepared to toss such ideas into the melting pot, throwing them out or revising them as observations require. The observers, on the other hand, seem to wish to build complete detailed models on the basis of incomplete data, while justifying the procedure with a barrage of statistics (not always valid for small samples) and the interchangeable, largeerror graphs which I have already mentioned.

Perhaps this is a result of the lack, in some cases, of astronomical training. With experiments which simply cannot be repeated and the everyday hazards of balloon and rocket observations, something more of the pioneering attitude seems to be needed in this last frontier of observational astronomy.

But on the other hand, this earnestness among the observers is really only manifested when they get up on their hind legs to address a gathering of

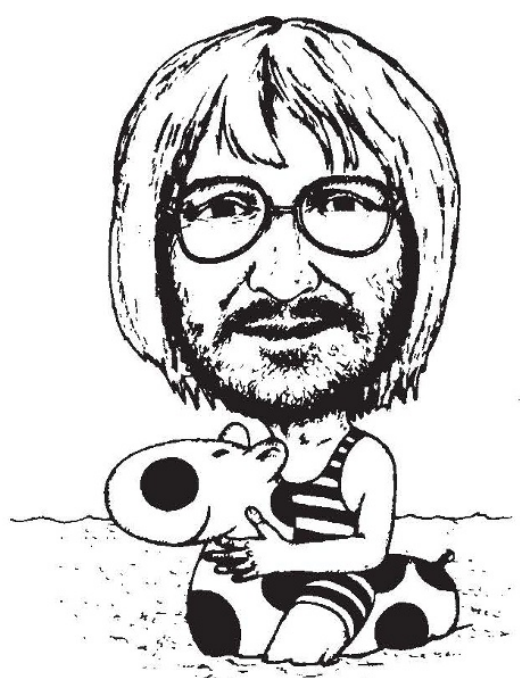

\section{Glaxo awards}

JонN Gribbin, of Nature, is among the four recipients of Glaxo Travelling Fellowships for science journalists announced on July 9. His award, in the National category, is chiefly for a series of articles on the significance of climatic change; much of this work has appeared in The Times Science Report and longer articles appeared in Nature, New Scientist and Environment and Change during 1973.

The awards, which are sponsored by Glaxo and administered in collaboration with the Association of British Science Writers, are in the form of $£ 500$ travel grants; John Gribbin will be using his award to travel to the United States and Canada during the autumn, and will be reporting for Nature on research into climatic
John Gribbin (left) lives by the sea at Brighton.

change being carried out there.

John Gribbin joined the staff of Nature in October 1970, after completing research for a $\mathrm{PhD}$ in astrophysics at the University of Cambridge. In that year, he received the First Award of the Gravity Research Foundation of New Boston for work on "Using Gravity to Determine the Nature of Superluminous Astronomical Objects"; for most of the past four years he has been in charge of the Nature-Times News Service, which reports on developments in all the sciences. As well as the interest in climatic change which has led to this award, John Gribbin is concerned about the application of science to other global problems, and is co-author of a book on earthquakes and earthquake prediction (The Jupiter Effect) which is to be published by Macmillan in September.

Fellowships in the other three categories (Radio and Television; Regional; and Trade, Technical and House Magazine) go to David Wilson (Science Correspondent of BBC Television News), Judith Hann, a freelance science journalist, and Geoffrey Watts, Deputy Editor of World Medicine. Miss Hann is the first person to win a Glaxo Fellowship for the second time; the first occasion was in 1967.

Last year's award winners included John Maddox, then Editor of Nature, in the National category. their peers. Individually or in small groups (especially after lubrication with the local Frascati wine) they appeared as intelligent people with the sense of humour needed to cope with the rigours of their trade. In some cases, they even agreed that their data were really rather vague and open to other interpretations, at least in detail

The symptoms are, indeed, very similar to those severe cases of jargonese which result when many people try to write a "scientific paper". In the long term, the answer to both problems must lie in a basic change of attitude in the direction of clarity and honesty of communication as opposed to cunning packaging which can only be interpreted by the initiated.

In the short term, since almost every scientist is willing to communicate as a human being on a face to face basis, saving the 'scientist' mask for lecturing, a solution might be to cut down on formal presentations at symposia, in favour of circulation of papers together with ample opportunity for informal discussion. At Frascati, more than 50 talks were on the official timetable (covering $2 \frac{1}{2}$ working days) and more were crammed in at short notice. Apart from the obvious benefits of a trip to Italy in June, it is difficult to see how the participants would have been less well served simply by reading the papers. The advantages of a symposium should be personal contact and the opportunity to assess the abilities of colleagues in the same field who may work on another continent; it seems that the time has certainly come when a move away from the present system, in the direction of the original "drinking party" implications of the word "symposium" could well be a good thing. 